\title{
Decrease of choriocapillary vascular density measured by optical coherence tomography angiography in Vogt-Koyanagi-Harada disease
}

\author{
Anyi Liang ${ }^{1}$. Shanshan $\mathrm{Jia}^{2} \cdot \mathrm{Fei} \mathrm{Gao}^{1} \cdot$ Xiaoxu Han ${ }^{1} \cdot$ Minghang Pei $^{3} \cdot \mathrm{Yi} \mathrm{Qu}^{4} \cdot \mathrm{Junyan}_{\mathrm{Xiao}}{ }^{1} \cdot$ Chan Zhao $^{1,5}$. \\ Meifen Zhang ${ }^{1,5}$
}

Received: 25 November 2020 / Revised: 1 April 2021 / Accepted: 5 May 2021 / Published online: 3 July 2021

(c) The Author(s) 2021

\begin{abstract}
Purpose Changes of choroidal circulation throughout the disease course of Vogt-Koyanagi-Harada (VKH) disease and the clinical significance remain unclear. Choriocapillary vascular density (CC VD) measured by optical coherence tomography angiography (OCTA) were compared in different disease stages of VKH and its correlation with other parameters was analyzed, aiming to explore their clinical relevance.

Methods This is a retrospective case series. One hundred and fourteen VKH patients and 47 normal controls (NCs) were included. Patients were grouped into the acute uveitic, convalescent, and chronic recurrent stages (only anterior recurrent cases included), and OCTA images were obtained from VKH patients in these stages. Best corrected visual acuity (BCVA), CC VD, and subfoveal choroidal thickness (SFCT) were recorded and compared.

Results CC VD in acute $(58.26 \% \pm 0.84 \%)$, convalescent $(64.85 \% \pm 0.33 \%)$, and chronic recurrent $(62.78 \% \pm 0.70 \%)$ stage of VKH patients were all significantly lower than that in NCs $(66.37 \% \pm 0.41 \%)(p<0.001, p=0.017$, and $p<0.001$, respectively). CC VD increased by $6.59 \% \pm 0.91 \%$ with resolution of acute inflammation $(p<0.001)$ and decreased by $2.07 \% \pm 0.74 \%$ during anterior uveitis relapse $(p=0.009)$. Patients with a positive history of anterior recurrence had lower CC VD $(-2.43 \% \pm 0.75 \%, p=0.003)$ in the convalescent stage than those without. CC VD was negatively correlated with $\log$ MAR BCVA in VKH $(r=-0.261, p<0.001)$.

Conclusion CC VD was decreased in every stage of VKH. CC VD has the potential to reflect the status of uveitis and might be promising in monitoring the disease activity. OCTA is a convenient and straightforward tool to evaluate choroidal vascularity, and CC VD provides supplemental quantitative information of the choriocapillaris. Further studies are needed to explore the values of OCTA quantitative parameters in monitoring VKH progression, predicting visual prognosis, and guiding clinical decisions.
\end{abstract}

Keywords Vogt-Koyanagi-Harada disease $\cdot$ Optical coherence tomography angiography $\cdot$ Choriocapillary vascular density $\cdot$ Disease stage $\cdot$ Visual acuity

Chan Zhao

zhaochan@pumch.cn

Meifen Zhang

meifen_zhang@ @otmail.com

1 Department of Ophthalmology, Peking Union Medical College Hospital (Dongdan Campus), Chinese Academy of Medical Sciences, No.1 Shuaifuyuan Wangfujing Dongcheng District, Beijing 100730, China

2 Department of Ophthalmology, The Second Clinical Medical College (Shenzhen People's Hospital), Jinan University, Shenzhen 518020, China
3 Department of Ophthalmology, First Affiliated Hospital of Zhengzhou University, Zhengzhou 450052, Henan, China

4 Department of Ophthalmology, Peking University Third Hospital, Beijing 100191, China

5 Key Laboratory of Ocular Fundus Diseases, Chinese Academy of Medical Sciences, Beijing, China 


\section{Key messages}

- Choroidal circulation was observed to be impaired in acute and chronic VKH patients in previous studies, but not quantified or followed up throughout the disease course;

- This paper revealed that choroidal circulation was impaired in every stage of VKH. CC VD was negatively correlated with $\log$ MAR BCVA;

- CC VD provides supplemental quantitative information of the choriocapillaris. It has the potential to reflect the status of uveitis and might be promising to be used to monitor the disease activity;

- OCTA is a convenient and straightforward tool to evaluate choroidal vascularity.

\section{Introduction}

Vogt-Koyanagi-Harada (VKH) disease is a T cell-mediated multisystemic, granulomatous autoimmune disorder with ocular involvement in the form of bilateral granulomatous panuveitis [1]. Repeated episodes of uveitis may cause severe complications and poor visual outcome [2-7]. Notably, with the help of imaging modalities including enhanced depth imaging optical coherence tomography (EDI-OCT) [8, 9] and indocyanine green angiography (ICGA) [10], choroidal changes of VKH can be viewed and partially quantified.

Advances in optical coherence tomography angiography (OCTA) have allowed in vivo visualization and quantitative measurement of retinal and choroidal vasculatures with high repeatability and reproducibility. In the area of uveitis, OCTA is becoming increasingly important not only as a diagnostic imaging modality supplementing traditional techniques, but also as a means to provide quantitative evaluations during follow-up [11]. Choriocapillary (CC) blood flow and vascular densities (VD) were found to be significantly decreased in both acute and chronic VKH patients in several recent studies of ours and other teams [12-14], indicating substantial choroidal circulation impairment. But how the choroidal blood flow changes throughout the disease course and whether there is any clinical significance remain unclear, and there have been very few studies on these topics.

To address this, we used OCTA to measure and compare CC VD in different disease stages of VKH and analyzed its correlation with other parameters, aiming to explore their clinical relevance.

\section{Materials and methods}

\section{Study participants}

This is a retrospective case series. Our study was approved by the Institutional Review Board of Peking Union Medical College Hospital (PUMCH) and followed the tenets of
Declaration of Helsinki. Informed consents were obtained from all participants for using their clinical data. OCTA images and medical records of VKH patients who underwent OCTA scans in PUMCH from October 2015 to December 2019 and normal controls (NC) were analyzed. Eyes with refractive errors greater than 6.00 diopters and other confounding chorioretinal pathologies were excluded. The diagnosis of VKH disease was based on the American Uveitis Society's revised international criteria [15], which was applied to each patient. Participants were grouped into the acute uveitic stage, convalescent or chronic stage, and chronic recurrent stage according to the classic Moorthy criteria [16].

\section{Measurements of vascular densities by OCTA}

OCTA images were obtained with AngioVue (Optovue, Fremont, CA, USA). Segmentation and quantification of the vascular plexus were performed automatically by the built-in software. Serous retinal detachment and choroidal folds are common in the acute stage, which cause difficulties and compromise accuracy in the measurement of CC VD by introducing errors in segmentation of the choriocapillaris. So, every OCTA image was proofread and manually edited with the built-in "Segmentation editing and automatic propagation" function of the device to improve accuracy when necessary (Fig. 1). The built-in "followup" program was used to ensure that the same OCTA sections were obtained in repeated examinations. Qualities of the images were graded automatically by the device as 10 levels, from Q1 (1/10, the worst) to Q10 (10/10, the best), and only scans with qualities of Q6 or above were included in this study.

Scans from eyes with active (either at the acute uveitic or the anterior uveitis recurrent stage) or inactive (at the convalescent stage) inflammation were selected for analysis. Best corrected visual acuity (BCVA) in logMAR units (recorded as $\operatorname{logMAR}$ BCVA), choriocapillary vascular density (CC VD), and subfoveal choroidal thickness (SFCT) were recorded for all participants. CC VD was measured in the "macular, $3 * 3 \mathrm{~mm}$ " scanning mode. $\mathrm{CC}$ was defined as from $10 \mu \mathrm{m}$ above Bruch's membrane (BRM) to $30 \mu \mathrm{m}$ below BRM and CC VD was 

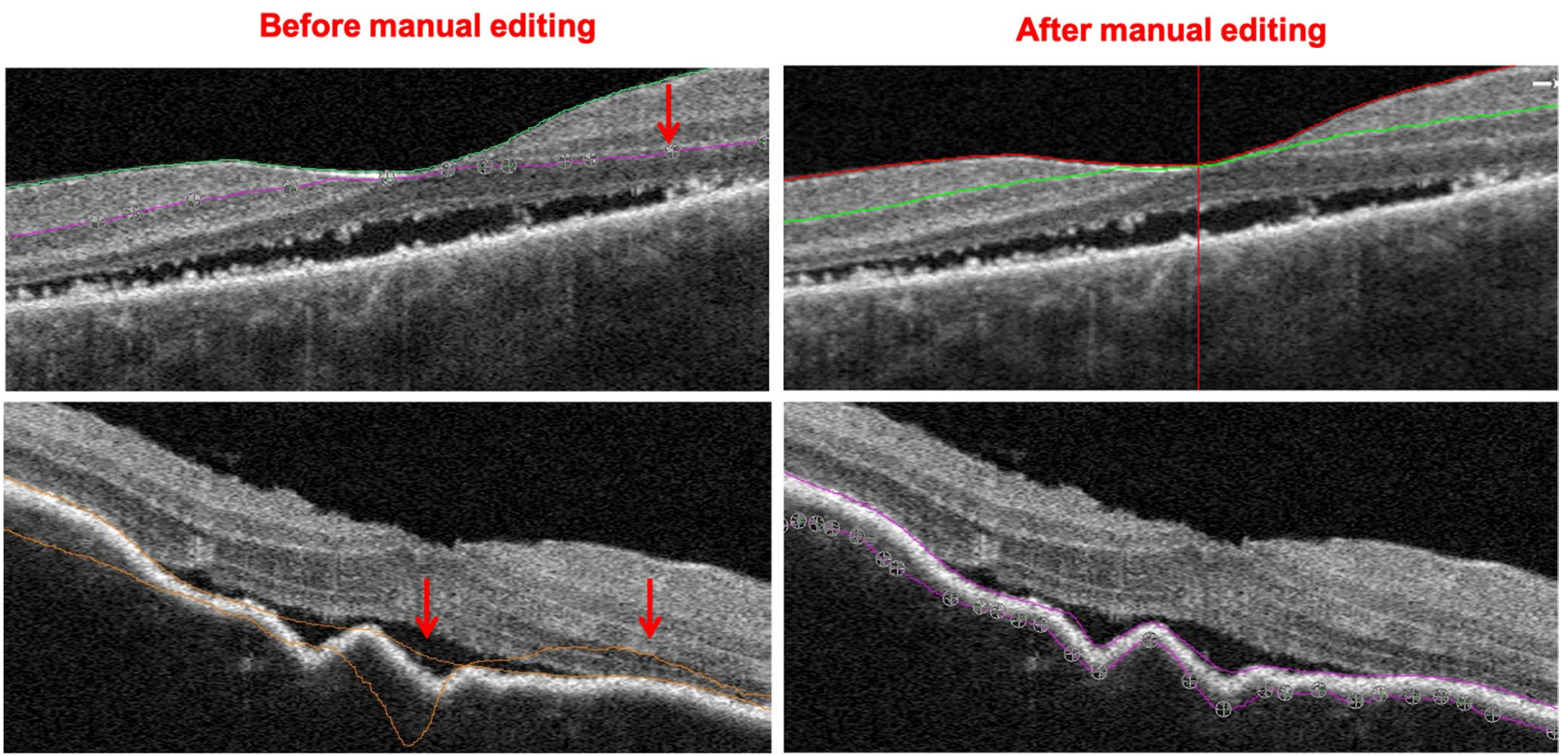

Fig. 1 Pictures demonstrating the segmentation before and after manual correction. Red arrows: locations of erroneous segmentation

calculated as the percentage of flow area (FA) over selected area (SA) (illustrated in Fig. 2). SFCT was measured under the fovea from the outer border of the retinal pigment epithelium (RPE) to the inner border of sclera (illustrated in Fig. 3). For choroidal thickness that was beyond measurement limit of the OCTA system, $800 \mu \mathrm{m}$ was recorded as the value according to the strategies of previous studies $[8,17,18]$.

\section{Statistical analysis}

IBM SPSS Statistics (Armonk, NY, IBM Corp.) version 22.0 and GraphPad Prism ${ }^{\circledR}$ (GraphPad Software Inc., La Jolla, CA) version 6.01 were used for statistical analysis and graphs presentation, respectively. A $p$ value equal to or less than 0.05 was considered significant for all statistical testing. CC VD and SFCT changes during the disease course and between patients and controls were performed using nonparametric test. The correlations between VD and other parameters were analyzed with Pearson correlation coefficient.

\section{Results}

\section{Study participants}

A total of $114 \mathrm{VKH}$ patients (52 males, 62 females, 217 eyes) and 47 age-matched NCs (24 males, 23 females, 94 eyes) were enrolled in the study. Eleven eyes from 11 patients were excluded because the quality of their OCTA images was poor. The mean ages of $\mathrm{VKH}$ patients and $\mathrm{NCs}$ were $41.0 \pm 1.3$ years $(9-69$ years $)$ and $41.2 \pm 1.8$ years (14-64 years), respectively $(p=0.956)$. Ninety-four OCTA scans from NCs and 726 from VKH patients were reviewed and analyzed, among which 135 (18.6\%) were obtained in the acute stage, $540(74.4 \%)$ in the convalescent stage, and $51(7.0 \%)$ in the chronic anterior recurrent stage. For patients who had more than 1 OCTA scans in the same stage, quantitative measurements of images from different time points in that stage were averaged.

\section{Choriocapillary vascular densities and choroidal thickness in VKH and NCs}

CC VD was $62.64 \% \pm 0.36 \%$ in VKH patients (regardless of stages), which was significantly lower than that in NCs $(66.37 \% \pm 0.41 \%, p<0.001)$. SFCT was $366.69 \mu \mathrm{m} \pm 9.15 \mu \mathrm{m}$ in VKH patients (regardless of stages), which was significantly thicker than that in NCs (218.30 $\mu \mathrm{m} \pm 6.91 \mu \mathrm{m}, p<0.001)$ (Fig. 4).

Representative images of choriocapillary layer with CC VD measurements in different stages of VKH are shown in Fig. 5.

CC VD, SFCT, and LogMAR BCVA in different stages of VKH patients and in NCs are shown in Table 1. Compared to NCs, CC VD was significantly decreased in the acute stage $(-8.11 \% \pm 0.94 \%$; $95 \%$ CI: $-6.25 \%,-9.97 \%$; $p<0.001)$, convalescent stage $(-1.52 \% \pm 0.54 \%$; $95 \%$ CI: $-2.59 \%,-0.45 \% ; p=0.017)$, and chronic anterior recurrent stage $(-3.59 \% \pm 0.77 \%$; $95 \% \mathrm{CI}:-5.10 \%,-2.07 \%$; $p<0.001)$; SFCT was significantly increased in the acute stage (increased by $278.81 \mu \mathrm{m} \pm 20.33 \mu \mathrm{m}$; 95\% CI: 
Fig. 2 Illustration of choriocapillary vascular density measurement. Top: segmentation standard. Area between 2 red lines stands for choriocapillaris. Bottom: vascular density measurement. The total area of the yellow circle is the selected area $\left(\mathrm{SA}, \mathrm{mm}^{2}\right)$; signals highlighted in yellow within the circle stands for blood flow area $\left(\mathrm{FA}, \mathrm{mm}^{2}\right)$; black within the circle indicates area without blood flow signal. CC VD is calculated as FA/SA (\%). BRM: Bruch's membrane

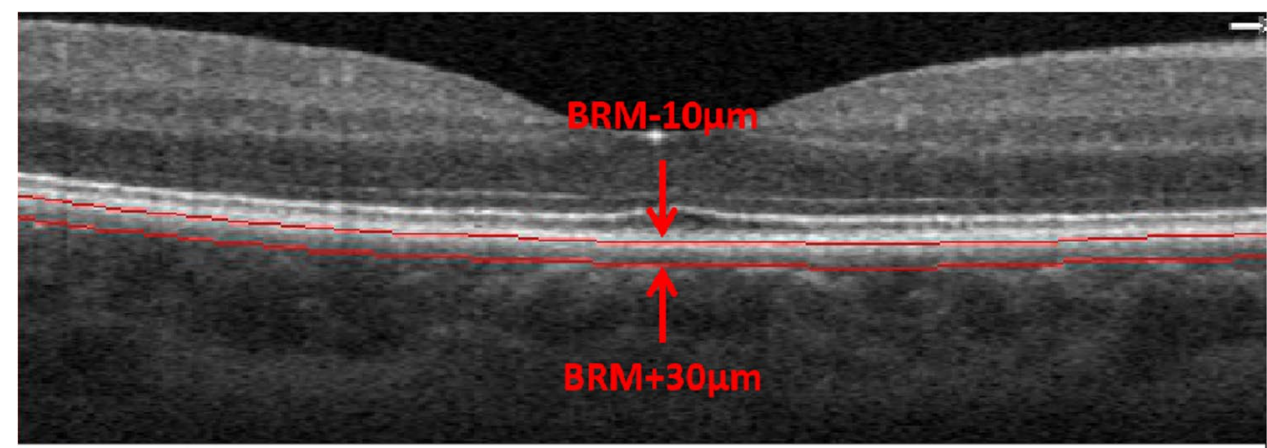

Angio Retina

Select Area $\left(\mathrm{mm}^{2}\right): 3.144$ Flow Area $\left(\mathrm{mm}^{2}\right)=2.309$

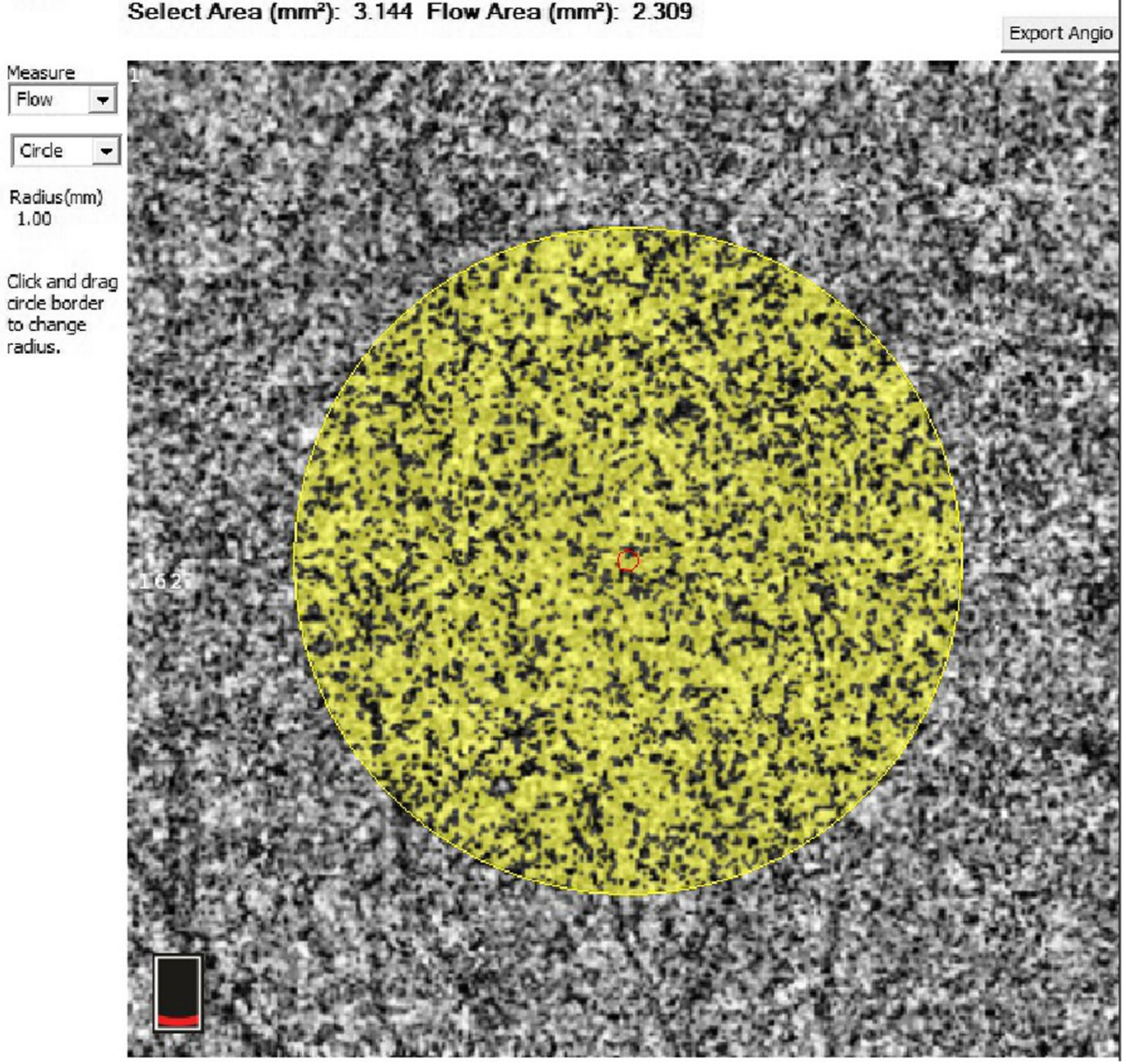

$238.54 \mu \mathrm{m}, 319.09 \mu \mathrm{m} ; p<0.001)$, convalescent stage (increased by $81.51 \mu \mathrm{m} \pm 10.83 \mu \mathrm{m}$; 95\% CI: $60.19 \mu \mathrm{m}$, $102.83 \mu \mathrm{m} ; p<0.001$ ), and chronic anterior recurrent stage (increased by $146.19 \mu \mathrm{m} \pm 19.33 \mu \mathrm{m}$; 95\% CI: $107.48 \mu \mathrm{m}$, $184.90 \mu \mathrm{m} ; p<0.001)$ compared to the NC.

\section{Changes of vascular densities, choroidal thickness, and visual acuity over different VKH stages}

Figure 6 showed the dynamic changes of CC VD, SFCT, and LogMAR BCVA over different VKH stages. With resolution of acute inflammation, CC VD increased by $6.59 \% \pm 0.91 \%$ (95\% CI: $4.08 \%$, $8.39 \% ; p<0.001)$; SFCT decreased by $197.31 \mu \mathrm{m} \pm 20.85 \mu \mathrm{m}(95 \%$ CI: $156.03 \mu \mathrm{m}, 238.58 \mu \mathrm{m} ; p<0.001)$; LogMAR BCVA decreased by $0.21 \pm 0.04$ (95\% CI: $0.14,0.29 ; p<0.001)$. During anterior uveitis relapse, CC VD decreased by $2.07 \% \pm 0.74 \%$ (95\% CI: $0.62 \%$, $3.52 \% ; p=0.009)$; SFCT increased by $64.68 \mu \mathrm{m} \pm 18.78 \mu \mathrm{m}(95 \%$ CI: $27.67 \mu \mathrm{m}, 101.70 \mu \mathrm{m} ; p=0.001) ; \operatorname{logMAR}$ BCVA increased by $0.08 \pm 0.04$ (95\% CI: $0.00,0.16 ; p=0.016$ ).

\section{Comparison between patients with and without histories of anterior uveitis recurrence}

Among the $114 \mathrm{VKH}$ patients included in this study, 63 had been followed up for more than 1 year and were further grouped into with or without a history of anterior uveitis recurrence. OCTA scans of these patients obtained in the 


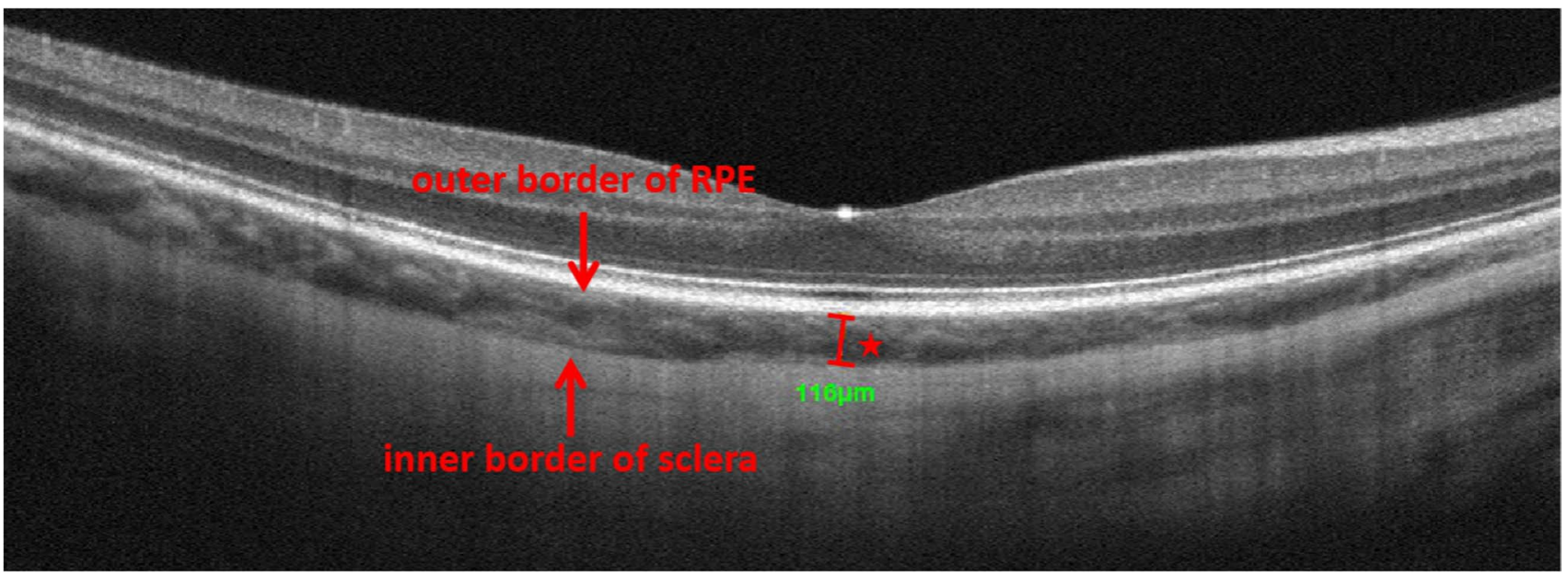

Fig. 3 Illustration of subfoveal choroidal thickness measurement. SFCT is measured as the vertical distance indicated by the red star. RPE: retinal pigment epithelium

convalescent stage were analyzed. Results showed that compared to patients who never had anterior recurrences during the disease course $(n=30)$, those who had a positive history of anterior recurrence $(n=33)$ had lower CC VD $(-2.43 \% \pm 0.75 \%, p=0.003)$, while the differences of SFCT $(-1.03 \mu \mathrm{m} \pm 20.23 \mu \mathrm{m}, p=0.97)$ and $\log$ MAR BCVA $(0.05 \pm 0.03, p=0.102)$ were not statistically significant (Table 2).

\section{Correlation between vascular density, choroidal thickness, and visual acuity}

Correlation analysis revealed that $\mathrm{CC}$ VD was negatively correlated with $\log$ MAR BCVA in VKH. When analyzed in different stages separately, CC VD was negatively correlated with $\log$ MAR BCVA only in the acute uveitic stage, but not in other stages. SFCT was not significantly correlated with logMAR BCVA in any stage. CC VD appeared to be negatively correlated with SFCT (Table 3).
Fig. 4 Comparisons of $\mathrm{CC}$ VD and SFCT between VKH and NCs. CC VD decreased and SFCT increased in VKH patients compared to NCs
Comparison of CC VD

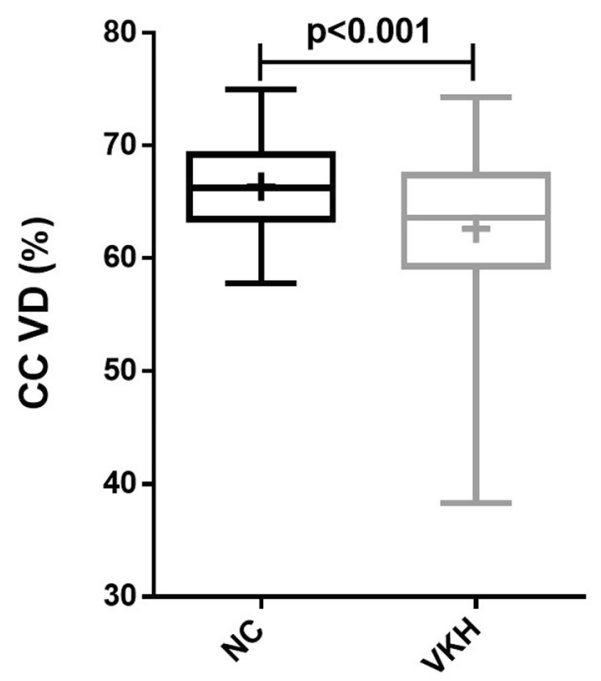

Group
Comparison of SFCT

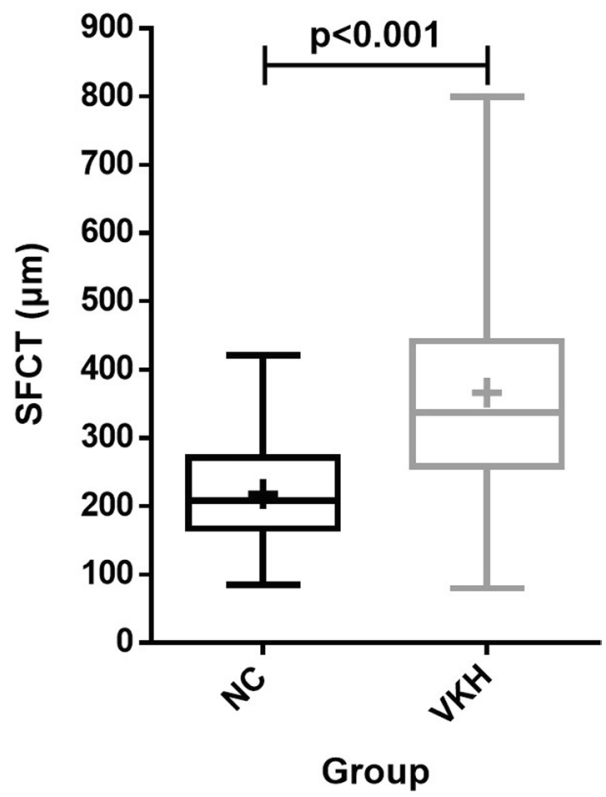




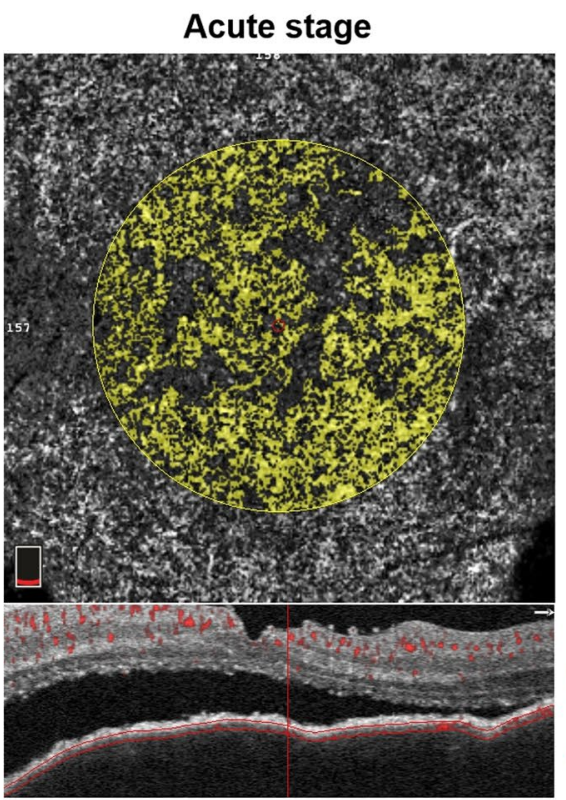

Selected Area $\left(\mathrm{mm}^{2}\right): \mathbf{3 . 1 4 4}$

Flow Area $\left(\mathrm{mm}^{2}\right): 1.405$ CC VD: $44.7 \%$

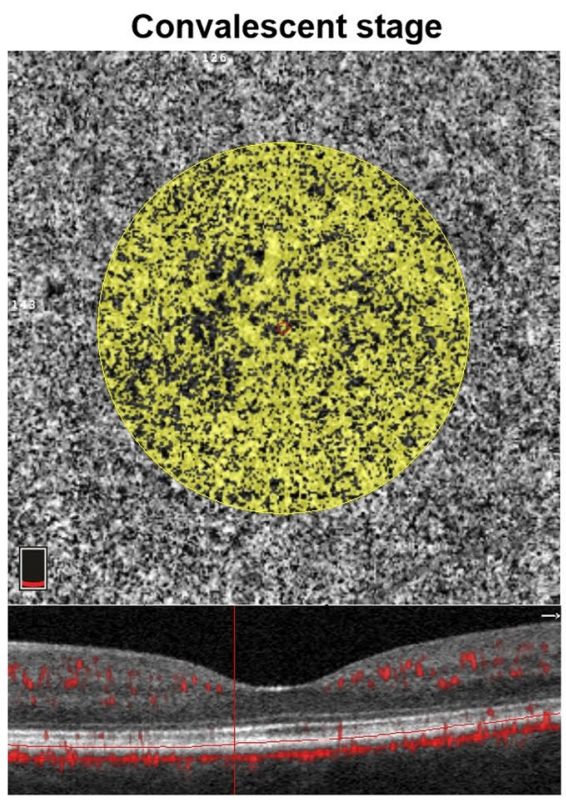

Selected Area $\left(\mathrm{mm}^{2}\right): 3.144$

Flow Area $\left(\mathrm{mm}^{2}\right): \mathbf{2 . 1 7 5}$ CC VD: $69.2 \%$

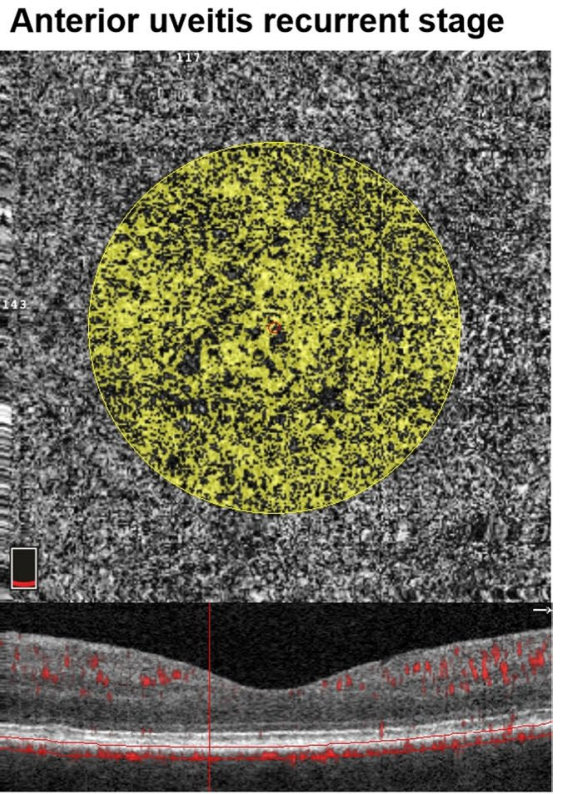

Selected Area $\left(\mathrm{mm}^{2}\right): 3.144$

Flow Area $\left(\mathrm{mm}^{2}\right)$ : 1.916 CC VD: $60.9 \%$

Fig. 5 Demonstration of representative images of CC VD measurement (from the same eye of the same patient)

\section{Discussion}

To the best of our knowledge, there have been few researches on the quantitative analysis of choroidal circulation in $\mathrm{VKH}$ so far. This is the first study using OCTA to investigate choroidal blood flow changes in different stages of VKH. Furthermore, we are pioneering in analyzing the correlation between vascular density and thickness of the choroid and their correlation with vision. Besides, the sample size in this study was relatively large.

Choroid is the main site of ocular inflammation in VKH disease [1], so characterization of the pathophysiological features of the choroid would be of great significance to the diagnosis, follow-up, and evaluation of treatment effectiveness. Morphological changes are mostly straightforward and apparent, but quantification analysis would be more accurate in reflecting the severity and changes of inflammation. Choroidal thickness (CT) and blood circulation are the 2 main quantification indexes. Most of the reports about CT are consistent with our study, where we detected a dramatically thickened choroid in the acute uveitic, convalescent, and anterior uveitic recurrent stages of VKH. The CT was reported to increase in the acute phase [19], subside with effective treatment [17], and rebound with uveitis recurrence [18] in previous studies, the same changing mode as that in our study. These findings indicated that thickening of the choroid is one of the hallmarks of VKH. CT can be used as a quantifiable marker of disease severity as it changes sensitively with the aggravation or resolution of inflammation.

ICGA is a classic and valuable tool to evaluate vessel conditions in the choroid, which can reflect choroidal hypoperfusion and vascular leakages in VKH [20]. It can be used to semi-quantify and evaluate the choroidal

Table 1 CC VD, SFCT, and LogMAR BCVA in VKH and NCs

\begin{tabular}{lllll}
\hline Parameters & Acute uveitic stage $(n=90)$ & $\begin{array}{l}\text { Convalescent stage } \\
(n=175)\end{array}$ & $\begin{array}{l}\text { Chronic anterior recurrent } \\
\text { stage }(n=46)\end{array}$ & Normal control $(n=94)$ \\
\hline CC VD $(\%)$ & $58.26 \pm 0.84$ & $64.85 \pm 0.33$ & $62.78 \pm 0.70$ & $66.37 \pm 0.41$ \\
& $(38.3,72.8)$ & $(54.7,74.3)$ & $(53.4,72.7)$ & $(57.8,75.0)$ \\
SFCT $(\mu \mathrm{m})$ & $497.11 \pm 19.12$ & $299.80 \pm 8.33$ & $364.49 \pm 18.06$ & $218.30 \pm 6.91$ \\
& $(163,800)$ & $(80,690)$ & $(162,642)$ & $(85,421)$ \\
logMAR BCVA & $0.32 \pm 0.03$ & $0.11 \pm 0.02$ & $0.19 \pm 0.04$ & NA \\
& $(-0.13,1.10)$ & $(-0.10,1.15)$ & $(0.00,1.00)$ & \\
\hline
\end{tabular}

All the values were presented as mean \pm standard error (minimum, maximum) 
Changes of CC VD

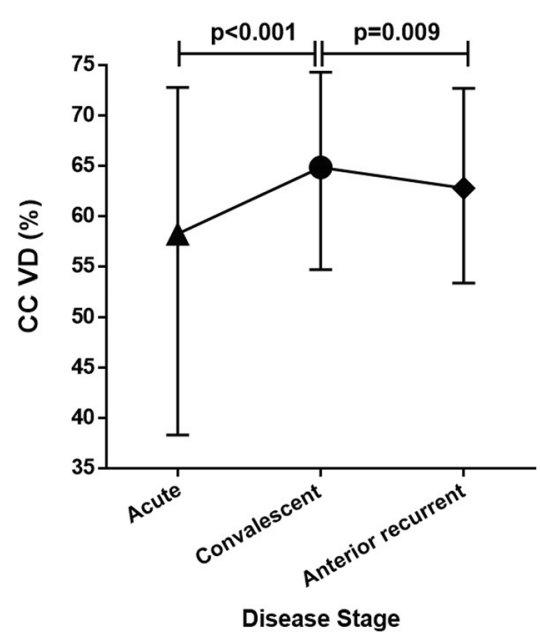

Changes of SFCT

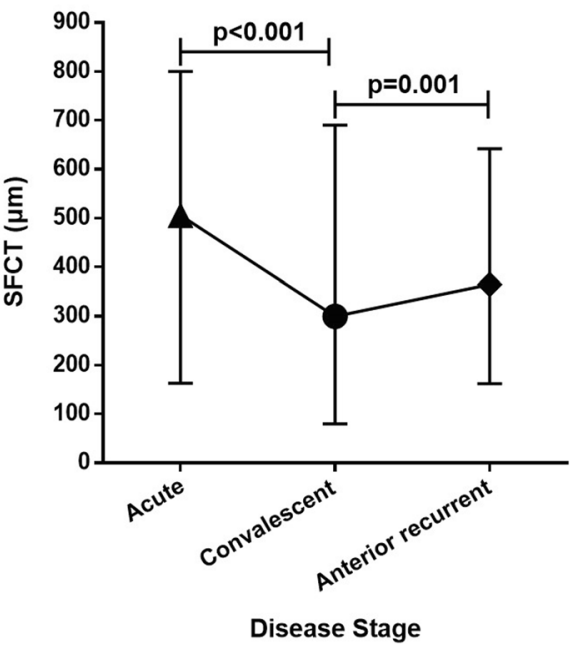

Changes of BCVA

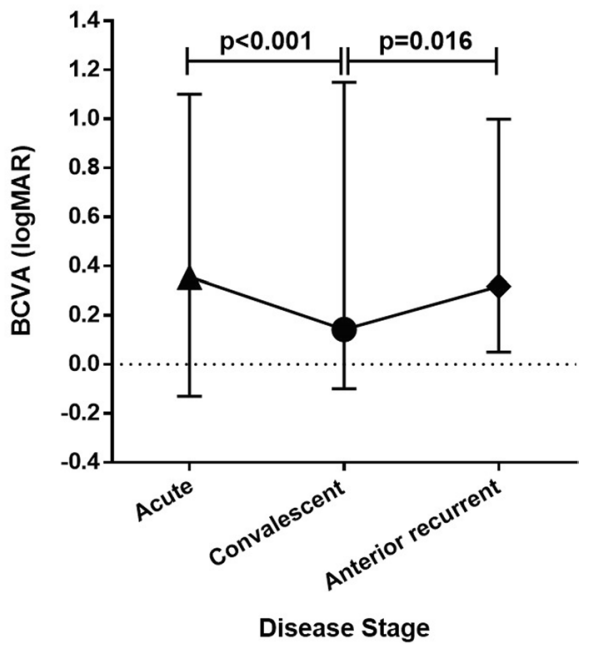

Fig. 6 Dynamic changes of CC VD, SFCT, and LogMAR BCVA over different disease stages of VKH. CC VD increased with uveitis resolution and decreased during anterior uveitis recurrence. SFCT

blood flow by calculating the number and total area of hypofluorescent dark dots (HDDs) [10]. Combined with FFA, ICGA can also quantify the severity of uveitis by scoring the signs of uveitis [21]. However, because of its invasiveness, dye-related complications, and inability to directly quantify vascular densities accurately, other techniques were used to supplement the functions of ICGA. Decrease of choroidal vascularity index (CVI) and blood flow velocity in active VKH were detected with EDI-OCT and laser speckle flowgraphy (LSFG), respectively [10], indicating circulation impairment of the choroid. In this study, we adopted the latest blood flow quantification method, OCTA, to evaluate the circulation, which is more convenient and straightforward. OCTA cannot detect the signals of flow velocity below a certain limit [22], i.e., too slow or static, so what was measured might reflect the actual effective circulatory amount, which makes the measurement more meaningful. In contrast, in Liu et al.'s decreased with uveitis resolution and increased during anterior uveitis recurrence. LogMAR BCVA decreased with uveitis resolution and increased during anterior uveitis recurrence

study [9], CVI was higher in VKH than that in NC even though choroidal circulation was impaired, because the increased blood in choroid might be static.

CC VD of VKH was found to be lower than that of $\mathrm{NC}$ in every disease stage, indicating choroidal circulation abnormality throughout the whole disease course. The impairment of choroidal blood flow was confirmed in various studies with different techniques, including hypofluorescent dark dot and decreased dye filling velocity of the choroid on ICGA [10, 23], decreased choroidal blood flow velocity on LSFG, decreased CVI on EDI-OCT [9], and lower CC VD on OCTA [12]. Notably, we noticed that even in the chronic convalescent stage when inflammation was inactive, CC VD was still lower than that of NC. One of the reasons might be that damages to the choroidal blood flow by inflammation were persistent and partially irreversible. Another possible explanation is that even the uveitis appeared to be inactive clinically, potential subclinical inflammatory activity
Table 2 Comparison between patients with and without histories of anterior uveitis recurrence

\begin{tabular}{lllll}
\hline $\begin{array}{l}\text { History of anterior } \\
\text { uveitis recurrence }\end{array}$ & $\begin{array}{l}\text { Number of } \\
\text { patients }\end{array}$ & CC VD $(\%)$ & SFCT $(\mu \mathrm{m})$ & LogMAR BCVA \\
\hline Positive & 33 & $\begin{array}{l}63.72 \pm 4.35 \\
(54.8,71.8)\end{array}$ & $\begin{array}{l}309.92 \pm 109.98 \\
(124,571)\end{array}$ & $\begin{array}{l}0.08 \pm 0.19 \\
(-0.09,0.98)\end{array}$ \\
Negative & 30 & $66.06 \pm 3.65$ & $310.95 \pm 108.85$ & $0.03 \pm 0.13$ \\
& & $(54.8,72.4)$ & $(85,690)$ & $(-0.10,0.70)$ \\
Difference & NA & $-2.43 \pm 0.75$ & $-1.03 \pm 20.23$ & $0.05 \pm 0.03$ \\
& & $(-3.82,-0.87)$ & $(-41.11,39.04)$ & $(-0.02,0.11)$ \\
$p$ value & NA & $\mathbf{0 . 0 0 3}$ & 0.97 & 0.102 \\
\hline
\end{tabular}

All the values were presented as mean \pm standard error (minimum, maximum)

The bold data mean that the $p$ values are significant $(<0.05)$ 
Table 3 Correlation analyses between CC VD, SFCT, and logMAR BCVA in VKH patients

\begin{tabular}{|c|c|c|c|c|c|c|c|c|c|}
\hline \multirow[t]{2}{*}{ Stage } & & \multicolumn{2}{|l|}{3 stages } & \multicolumn{2}{|l|}{ Acute } & \multicolumn{2}{|c|}{ Convalescent } & \multicolumn{2}{|c|}{ Anterior recurrent } \\
\hline & & CC VD & SFCT & CC VD & SFCT & CC VD & SFCT & CC VD & SFCT \\
\hline \multirow[t]{2}{*}{ BCVA } & $r$ & -0.261 & 0.096 & -0.244 & -0.19 & -0.046 & -0.093 & 0.059 & -0.068 \\
\hline & $p$ & $<0.001$ & 0.124 & 0.036 & 0.103 & 0.594 & 0.276 & 0.708 & 0.67 \\
\hline \multirow[t]{2}{*}{ CC VD } & $r$ & & -0.419 & & -0.326 & & 0.007 & & -0.142 \\
\hline & $p$ & & $<0.001$ & & 0.002 & & 0.923 & & 0.352 \\
\hline
\end{tabular}

The bold data mean that the $p$ values are significant $(<0.05)$ might exist and continue to damage the choroidal circulation. Nongranulomatous inflammatory cell infiltration and atrophy of the choriocapillaris during convalescent stage in pathological observation might also explain the circulation defect [24].

In our study, CC VD was found to significantly decrease in the acute uveitis stage, increase as the inflammation subsided, and drop again when uveitis relapsed, meaning that choroidal circulation changed in accordance with the inflammation. It has long been reported and accepted that SFCT also change sensitively with the inflammatory activity in VKH eyes. A number of researches reported that SFCT increased in active inflammation and recovered after treatment $[8,17]$. Tagawa $Y$ et al. [8] also observed choroid thickening 1 month prior to development of anterior segment inflammation. Correlation analysis revealed that CC VD was negatively correlated with SFCT, meaning that choroidal circulation was worse in thicker choroid in VKH. This might be explained by the pathological changes of the choroid in VKH [24-26]. Large numbers of inflammatory cells infiltrating the choroidal stroma led to stromal edema, which compressed the choriocapillaris and caused blood circulation impairment. The consistency of changing modes of these 2 indexes and their correlation indicated that CC VD might be a novel quantitative marker to evaluate VKH patients.

We discovered that in the anterior uveitis recurrent stage, even if the relapse was in the anterior segment and no inflammatory signs were detected in the fundus, the CC VD was still found to be decreased. It indicated that there might be potential inflammation of posterior segment in anterior uveitis recurrence. Multiple lineages of evidences have revealed that the posterior segment was concomitantly inflamed during anterior uveitis recurrences in VKH patients. Chee SP and colleagues [27] reported that in a series of $\mathrm{VKH}$ patients with clinically isolated anterior uveitis recurrence, ICGA revealed early large choroidal vessel leakage and late diffuse leakage, which subsided after intensive immunosuppressive treatment. Using ICGA and LSFG, Kenichi Namba and colleagues [10] later demonstrated subclinical choroidal circulation impairment due to granulomatous inflammation as well as post-treatment improvement in isolated anterior uveitis recurrence in VKH. And this was also evidenced by our finding that SFCT increased in the anterior uveitis recurrent stage.
Although anterior uveitis recurrence is common in the chronic stage of VKH, not every patient would experience recurrent uveitis attacks. In order to find out whether there are any differences between patients with and without anterior recurrence in the chronic convalescent stage and whether these different presentations predict relapse or not, we compared CC VD, SFCT, and BCVA of these 2 groups of patients. Our results showed that patients with a positive history of anterior uveitis recurrence appeared to have lower CC VD than those without. Our hypothesis of this finding is that patients with a history of anterior recurrence had more severe ocular inflammation in the acute phase or less well-controlled disease in the chronic phase, which had resulted in more prominent and prolonged damages to the choroidal vasculature. However, further studies are needed to investigate whether anterior uveitis recurs as a result of CC VD impairment or vice versa.

BCVA is one of the main indicators of visual function. Quantitative OCTA measurements have been reported to be associated with visual outcome in a variety of diseases including diabetic retinopathy, retinal vein occlusion, and glaucoma [15, 28-31]. Similar findings were also reported in Behcet's uveitis by Cheng D et al. [32] that decreased deep retinal capillary plexus (DCP) VD was found to be correlated with reduced BCVA and disruption of outer retina substructures. So, it is worth exploring whether quantitative measurements by OCTA were correlated with VA or not in VKH. Our study revealed significant negative correlation between CC VD and logMAR BCVA, indicating that choroid quantitative parameters like VD might have the potential to reflect visual function. This result was not beyond expectation because CC VD reflects the perfusion condition of the choroid, which provides blood and metabolic supply for the outer retina. On the other hand, choroidal thickness did not appear to be correlated to visual acuity, indicating that CT was not an independent predictor of VA. Noticeably, visual function was influenced by a number of factors in the diseased state, including refractive media opacity, macular edema, and exudative retinal detachment, so further explorations are needed to find out the influencing factors of visual function in $\mathrm{VKH}$.

The following limitations should be kept in mind to interpret results of the current study: (1) the study was retrospective; thus, biases may exist. (2) Only the CC VD within 
$3 \mathrm{~mm}$ around the fovea was measured, which cannot represent the vasculature more peripherally. (3) The inherent motion and projection artifacts of OCTA may impair the accuracy of data.

\section{Conclusion}

In conclusion, this study confirmed that choroidal circulation was impaired in every stage of VKH. CC VD has the potential to reflect the status of uveitis and might be promising to be used to monitor the disease activity. OCTA is a convenient and straightforward tool to evaluate choroidal vascularity, and CC VD provides supplemental quantitative information of the choriocapillaris. Further studies are needed to explore the values of OCTA quantitative parameters in monitoring $\mathrm{VKH}$ progression, predicting visual prognosis, and guiding clinical decisions.

Authors' contributions AYL wrote the main manuscript text and prepared all figures and tables. SSJ, MHP, YQ, and JYX performed OCTA scans and helped data collections. MFZ and CZ assisted in the manuscript writing. MFZ, CZ, FG, and XXH performed ophthalmic examinations and clinical assessments of patients.

Funding This work is supported by the Non-profit Central Research Institute Fund of Chinese Academy of Medical Sciences (2018PT32029).

\section{Declarations}

Ethical approval This study was approved by the Institutional Review Board of Peking Union Medical College Hospital and conformed to the tenets of the Declaration of Helsinki.

Conflict of interest The authors declare no competing interests.

Open Access This article is licensed under a Creative Commons Attribution 4.0 International License, which permits use, sharing, adaptation, distribution and reproduction in any medium or format, as long as you give appropriate credit to the original author(s) and the source, provide a link to the Creative Commons licence, and indicate if changes were made. The images or other third party material in this article are included in the article's Creative Commons licence, unless indicated otherwise in a credit line to the material. If material is not included in the article's Creative Commons licence and your intended use is not permitted by statutory regulation or exceeds the permitted use, you will need to obtain permission directly from the copyright holder. To view a copy of this licence, visit http://creativecommons.org/licenses/by/4.0/.

\section{References}

1. Baltmr A, Lightman S, Tomkins-Netzer O (2016) Vogt-Koyanagi-Harada syndrome - current perspectives. Clin Ophthalmol $10: 2345-2361$
2. Read RW, Rechodouni A, Butani N et al (2001) Complications and prognostic factors in Vogt-Koyanagi-Harada disease. Am J Ophthalmol 131:599-606

3. Keino H, Goto H, Usui M (2002) Sunset glow fundus in VogtKoyanagi-Harada disease with or without chronic ocular inflammation. Graefes Arch Clin Exp Ophthalmol 240:878-882

4. Sheu SJ, Kou HK, Chen JF (2003) Prognostic factors for VogtKoyanagi-Harada disease. J Chin Med Assoc 66:148-154

5. Al-Kharashi AS, Aldibhi H, Al-Fraykh H et al (2007) Prognostic factors in Vogt-Koyanagi-Harada disease. Int Ophthalmol 27:201-210

6. Jap A, Luu CD, Yeo I, Chee SP (2008) Correlation between peripapillary atrophy and corticosteroid therapy in patients with Vogt-Koyanagi-Harada disease. Eye (Lond) 22:240-245

7. Wu K, Zhang X, Su Y et al (2016) Clinical characteristics of inflammatory choroidal neovascularization in a Chinese population. Ocul Immunol Inflamm 24:261-267

8. Tagawa Y, Namba K, Mizuuchi K et al (2016) Choroidal thickening prior to anterior recurrence in patients with Vogt-Koyanagi-Harada disease. Br J Ophthalmol 100:473-477

9. Liu S, Du L, Zhou Q et al (2018) The choroidal vascularity index decreases and choroidal thickness increases in VogtKoyanagi-Harada disease patients during a recurrent anterior uveitis attack. Ocul Immunol Inflamm 26(8):1237-1243

10. Takemoto Y, Namba K, Mizuuchi K et al (2016) Choroidal circulation impairment during the anterior recurrence of VogtKoyanagi-Harada disease confirmed with indocyanine green angiography and laser speckle flowgraphy. Acta Ophthalmol 94:e629-e636

11. Dingerkus V, Munk MR, Brinkmann MP et al (2019) Optical coherence tomography angiography (OCTA) as a new diagnostic tool in uveitis. J Ophthalmic Inflamm Infect 9:10

12. Jia SS, Zhao C, Gong D et al (2017) Optical coherence tomography angiography of acute Vogt-Koyanagi-Harada disease. Zhonghua Yan Ke Za Zhi 53:735-739

13. Luo K, Cai H, Hu Y et al (2020) Distinguishing microvasculature features of Vogt-Koyanagi-Harada in patients in acute and convalescent phases using optical coherence tomography angiography. Ocul Immunol Inflamm Jan 3:1-7

14. Fan S, Lin D, Hu J et al (2020) Evaluation of microvasculature alterations in convalescent Vogt-Koyanagi-Harada disease using optical coherence tomography angiography. Eye (Lond) Oct 6

15. Read RW, Holland GN, Rao NA et al (2001) Revised diagnostic criteria for Vogt-Koyanagi-Harada disease: report of an international committee on nomenclature. Am J Ophthalmol 131:647-652

16. Moorthy RS, Inomata H, Rao NA (1995) Vogt-KoyanagiHarada syndrome. Surv Ophthalmol 39:265-292

17. Maruko I, Iida T, Sugano Y et al (2011) Subfoveal choroidal thickness after treatment of Vogt-Koyanagi-Harada disease. Retina 31:510-517

18. Nakai K, Gomi F, Ikuno Y et al (2012) Choroidal observations in Vogt-Koyanagi-Harada disease using high-penetration optical coherence tomography. Graefes Arch Clin Exp Ophthalmol 250:1089-1095

19. Fong AH, Li KK, Wong D (2011) Choroidal evaluation using enhanced depth imaging spectral-domain optical coherence tomography in Vogt-Koyanagi-Harada disease. Retina 31:502-509

20. Herbort CP, Mantovani A, Bouchenaki N (2007) Indocyanine green angiography in Vogt-Koyanagi-Harada disease: angiographic signs and utility in patient follow-up. Int Ophthalmol $27: 173-182$

21. Tugal-Tutkun I, Herbort CP, Khairallah M, Angiography scoring for uveitis working group (ASUWOG) (2010) scoring of dual fluorescein and ICG inflammatory angiographic signs for the 
grading of posterior segment inflammation (dual fluorescein and ICG angiographic scoring system for uveitis). Int Ophthalmol 30:539-552

22. de Carlo TE, Romano A, Waheed NK, Duker JS (2015) A review of optical coherence tomography angiography (OCTA). Int J Retina Vitreous 15(1):5

23. Mawatari Y, Hirata A, Fukushima M et al (2006) Choroidal dye filling velocity in patients with Vogt-Koyanagi-Harada disease. Graefes Arch Clin Exp Ophthalmol 244:1056-1059

24. Rao NA (2007) Pathology of Vogt-Koyanagi-Harada disease. Int Ophthalmol 27:81-85

25. Inomata H, Sakamoto T (1990) Immunohistochemical studies of Vogt-Koyanagi-Harada disease with sunset sky fundus. Curr Eye Res 9(Suppl):35-40

26. Oh H, Takagi H, Takagi $\mathrm{C}$ et al (1999) The potential angiogenic role of macrophages in the formation of choroidal neovascular membranes. Invest Ophthalmol Vis Sci 40:1891-1898

27. Bacsal K, Wen DS, Chee SP (2008) Concomitant choroidal inflammation during anterior segment recurrence in Vogt-Koyanagi-Harada disease. Am J Ophthalmol 145:480-486

28. CasselholmdeSalles M, Kvanta A, Amrén U et al (2016) Optical coherence tomography angiography in central retinal vein occlusion: correlation between the foveal avascular zone and visual acuity. Invest Ophthalmol Vis Sci 57:OCT242-246

29. Wakabayashi T, Sato T, Hara-Ueno C et al (2017) Retinal microvasculature and visual acuity in eyes with branch retinal vein occlusion: imaging analysis by optical coherence tomography angiography. Invest Ophthalmol Vis Sci 58:2087-2094

30. Dupas B, Minvielle W, Bonnin S et al (2018) Association between vessel density and visual acuity in patients with diabetic retinopathy and poorly controlled type 1 diabetes. JAMA Ophthalmol 136:721-728

31. Jeon SJ, Park HL, Park CK (2018) Effect of macular vascular density on central visual function and macular structure in glaucoma patients. Sci Rep 8:16009

32. Cheng D, Shen M, Zhuang X et al (2018) Inner retinal microvasculature damage correlates with outer retinal disruption during remission in Behçet's posterior uveitis by optical coherence tomography angiography. Invest Ophthalmol Vis Sci 59:1295-1304

Publisher's note Springer Nature remains neutral with regard to jurisdictional claims in published maps and institutional affiliations. 